\title{
Continuity of solutions of linear, degenerate elliptic equations
}

\author{
JANI ONNINEN AND XIAO ZHONG
}

\begin{abstract}
We consider the simplest form of a second order, linear, degenerate, elliptic equation with divergence structure in the plane. Under an integrability condition on the degenerate function, we prove that the solutions are continuous.
\end{abstract}

Mathematics Subject Classification (2000): $30 \mathrm{C60}$ (primary); 35J15, 35J70 (secondary).

\section{Introduction}

Let $\Omega$ be a domain in $\mathbb{R}^{2}$. We consider the second order, linear, elliptic equation

$$
\operatorname{div}(A(x) \nabla u(x))=0,
$$

where $A(x)=\left[a_{i j}(x)\right]_{i, j=1,2}$ is a symmetric matrix with measurable coefficients defined in $\Omega$. Let $\alpha: \Omega \rightarrow[1, \infty]$ be an almost everywhere finite measurable function. We assume that the maximum eigenvalue of the matrix $A(x)$ is bounded by 1 and the minimum eigenvalue by $[\alpha(x)]^{-1}$, that is,

$$
\frac{|\xi|^{2}}{\alpha(x)} \leqslant\langle A(x) \xi, \xi\rangle \leqslant|\xi|^{2}
$$

for all $\xi \in \mathbb{R}^{2}$ and for almost every $x \in \Omega$. We say that a function $u \in W_{\text {loc }}^{1,1}(\Omega)$ has finite energy provided

$$
\langle A \nabla u, \nabla u\rangle \in L_{\mathrm{loc}}^{1}(\Omega) .
$$

In what follows, we always take $A$ and $\alpha$ as defined above. A function $u \in W_{\text {loc }}^{1,1}(\Omega)$ is a weak solution of the equation (1.1) provided that it has finite energy and (1.1)

Onninen was supported by the National Science Foundation grant DMS-0400611 and Zhong by the Academy of Finland, project 207288. Onninen thanks the people at University of Jyväskylä for their hospitality during his stay in Summer 2005, where this research was conducted. The work was finished when both authors visited the University of Cincinnati in Fall, 2005. They thank the department for its hospitality. Zhong also thanks the Taft Research Fund for the financial support.

Received July 26, 2006; accepted in revised form January 19, 2007. 
holds in the sense of distribution, i.e.

$$
\int_{\Omega}\langle A \nabla u, \nabla \phi\rangle \mathrm{d} x=0
$$

for all $\phi \in C_{0}^{\infty}(\Omega)$. We refer to [21] for the detailed discussion on the notion of solutions and the weighted Sobolev spaces.

The equation (1.1) is uniformly elliptic [4], if the function $\alpha$ is essentially bounded in $\Omega$. In this case, Morrey $[11,12]$ proved that solutions are Hölder continuous. The study of the best Hölder continuity exponent originated with the work of Widman [22] and Meyers [10]. Finally the sharp exponent was obtained by Piccinini and Spagnolo [17].

In higher dimensions ( $\mathbb{R}^{n}, n \geqslant 3$ ), Hölder continuity of solutions in the uniformly elliptic case was settled in the late 1950's. This study goes back to the pioneering work of De Giorgi [1] and Nash [15]. It was further developed by Ladyzhenskaya and Ural'tseva [7] to cover a wide range of quasilinear elliptic equations. Hölder continuity also follows from the Harnack inequality, established in the case of linear elliptic equations by Moser [13, 14]. Later, Serrin [18] and Trudinger [20] extended the Harnack inequality, and hence also Hölder continuity, to the quasilinear equations.

On the contrary, when the equation (1.1) is degenerate, that is, when $\alpha(x)$ is unbounded, finding the optimal condition on $\alpha(x)$ which guarantees continuity of the solutions seems to be far from settled. In the planar case, the standing conjecture of De Giorgi [2] reads as:

Conjecture 1.1. [DE GiORGi [2]] Let $A$ and $\alpha$ be as above. Suppose that

$$
\int_{\Omega} \exp \sqrt{\alpha(x)} \mathrm{d} x<\infty .
$$

Then any weak solution of the equation (1.1) is continuous in $\Omega$.

Conjecture 1.1 is one of the several conjectures (Conjecture 4) raised by De Giorgi [2] in his talk in Lecce, 1995. He also raised interesting conjectures in higher dimensions. These conjectures are still open, and as far as we know, the best known result is due to Trudinger [21]. We refer to [3], and the references therein, for a discussion of these challenging problems.

Let us next discuss the known results concerning Conjecture 1.1. The best known result [3] reads as: if $\alpha(x) \leqslant \mu\left(\left|x-x_{\circ}\right|\right)$ in a ball $\mathbb{B}\left(x_{\circ}, r\right)$, compactly contained in $\Omega$, for a function $\mu$ satisfying

$$
\int_{0}^{r} \frac{1}{t \mu(t)} \mathrm{d} t=\infty,
$$

then any weak solution $u$ of the equation (1.1) is continuous at the point $x_{\circ}$. The condition (1.6) requires that the function $\mu$ has almost logarithmic growth. In fact, on the one hand, for any $c>0$, the function

$$
\alpha_{0}(x)=c \log \left(e+\frac{1}{|x|}\right)
$$


satisfies the above assumption. Thus any weak solution of the equation (1.1) with the degenerate function $\alpha_{0}$ is continuous at the origin. Comparing this with the conjecture we realize that the function $\alpha_{0}$ enjoys a much stronger integrability condition than (1.5). Namely,

$$
\int_{\mathbb{B}(0,1)} \exp \left(\lambda \alpha_{0}(x)\right) \mathrm{d} x<\infty
$$

for any $\lambda<2 / c$. On the other hand, for any $\varepsilon>0, c>0$, the function

$$
\alpha_{\varepsilon}(x)=c \log ^{1+\varepsilon}\left(e+\frac{1}{|x|}\right)
$$

fails to satisfy the requirement (1.6). Again, comparing this assumption with the conjecture, but this time we find that $\alpha_{\varepsilon}(x)$ satisfies the integrability condition (1.5) in Conjecture 1.1 for any $\varepsilon<1$ and $c>0$; even $\alpha_{1}(x)$ does when $c<4$. Thus, there is a huge gap between the known result and Conjecture 1.1.

The result mentioned above follows from two facts. First, any weak solution of equation (1.1) is weakly monotone, see Section 3 for the detailed discussion. Second, the weak solution has finite energy. Actually, one can show that if $\alpha(x)$ is exponentially integrable, that is, the function $\alpha$ satisfies the condition (1.7), for some $\lambda>0$, then the solutions are continuous, see Remark 3.3. In this case, the solutions of equation (1.1) has a tight connection with mappings with exponentially integrable distortion. We refer to $[6,16]$ for the continuity property of this class of mappings.

In this note, we prove the following result.

Theorem 1.2. Let $\Omega$ be a domain in $\mathbb{R}^{2}$ and $\alpha: \Omega \rightarrow[1, \infty)$ be a function such that

$$
K=\int_{\Omega} \exp (\lambda \sqrt{\alpha(x)}) \mathrm{d} x<\infty \quad \text { for some } \lambda>1 .
$$

Then any weak solution $u$ of the equation (1.1) with the structure condition (1.2) is continuous in $\Omega$. Moreover, we have the following modulus of continuity. Suppose that the ball $\mathbb{B}_{R}=\mathbb{B}\left(x_{\circ}, R\right)$ is compactly contained in $\Omega$. Then for $\beta \in(0, \lambda-1)$, we have

$$
|u(x)-u(y)| \leqslant C \log ^{-\beta / 2}\left(\frac{K}{\pi r^{2}}\right)\left(\int_{\mathbb{B}_{R}}\langle A(x) \nabla u, \nabla u\rangle \mathrm{d} x\right)^{1 / 2},
$$

whenever $x, y \in \mathbb{B}_{r}$ and $r<\frac{R^{2}}{e^{16} K}$. Here the constant $C$ depends on $\beta, \lambda, R$ and $K$.

Naturally we ask if Theorem 1.2 is still true under the assumption (1.8) with $\lambda \leqslant 1$. This seems to be a delicate question. Let us look closely at Conjecture 7 in [2] for this issue. Let $\Omega=\left\{x=\left(x_{1}, x_{2}\right) \in \mathbb{R}^{2}:|x|<1 / e\right\}$ and $\mathbb{A}, \mathbb{B}$ be subsets of $\Omega$ given by the rule

$$
\mathbb{A}=\left\{x \in \Omega: 2\left|x_{2}\right|>|x|\right\}, \quad \mathbb{B}=\left\{x \in \Omega: 2\left|x_{2}\right|<|x|\right\} .
$$


For any $\epsilon>0$, we define a function $\tau$ on $\Omega$ as follows

$$
\tau(x)= \begin{cases}1 & \text { if } x \in \mathbb{A} \\ |\log | x||^{-(2+\epsilon)} & \text { if } x \in \mathbb{B} .\end{cases}
$$

Then Conjecture 7 in [2] states that the equation (1.1) with $A(x)=\tau(x) I$ has discontinuous solutions. As we can see, in this case $A(x)$ satisfies the structure condition (1.2) with $\alpha(x)=|\log | x||^{2+\epsilon}$, therefore,

$$
\int_{\Omega} \exp (\lambda \sqrt{\alpha(x)}) \mathrm{d} x=\infty, \quad \text { for } \lambda>0
$$

and

$$
\int_{\Omega} \exp \left(\lambda \alpha(x)^{q}\right) \mathrm{d} x<\infty, \quad \text { for } q<1 /(2+\epsilon) \text { and } \lambda>0 .
$$

Thus a positive answer to the above conjecture only shows that the square root in the integrability condition (1.8) is sharp.

The proof of Theorem 1.2 is divided into two steps, formulated as Lemma 3.2 and Lemma 3.4 in Section 3. Lemma 3.2 shows that the oscillation of the solution $u$ is controlled by its energy. It follows from the integrability assumption (1.5) of $\alpha(x)$ and the fact that $u$ is weakly monotone. Unfortunately, Lemma 3.2 is not enough to show that $u$ is continuous. It only shows that the solution cannot be severely discontinuous. The key for us is to prove a decay estimate for the energy of $u$, a Morrey type estimate [12]. This improvement for the energy is stated in Lemma 3.4 and is enough to cancel the discontinuity part of the oscillation estimate mentioned above to prove the continuity of $u$. Here we require that the constant $\lambda$ involving in the integrability assumption (1.8) on $\alpha(x)$ be big enough to do the cancellation. As a matter of fact, $\alpha>1$ will do. Finally, we remark that this proof is restricted to the planar case as in the uniformly elliptic case, since the Morry type estimate of the energy is not enough to guarantee the continuity of solutions in the higher dimensions.

\section{An auxiliary lemma}

The proof of Theorem 1.2 strongly relies on an auxiliary inequality, which is formulated in Lemma 2.1. Although, the proof of this inequality is an elementary consequence of Jensen's inequality, it gives the sharp estimate. This sharp bound will play a crucial role in the proofs of Lemma 3.2 and Lemma 3.4.

We denote the surface measure of the unit sphere $\partial \mathbb{B}(0,1)$ by $\omega_{n-1}$. The notation

$$
f_{\partial \mathbb{B}(y, r)} h(x) \mathrm{d} \sigma=\frac{1}{\omega_{n-1} r^{n-1}} \int_{\partial \mathbb{B}(y, r)} h(x) \mathrm{d} \sigma
$$

stands for the integral average of $h$ over the sphere $\partial \mathbb{B}(y, r)$. 
Lemma 2.1. Let $q \geqslant 1$ and $\gamma>0$ be two given constants, and $\Omega$ be a domain in $\mathbb{R}^{n}$. Suppose that a function $\beta: \Omega \rightarrow[1, \infty)$ is exponentially integrable, precisely

$$
K=\int_{\Omega} \exp (\lambda \beta(x)) \mathrm{d} x<\infty, \quad \text { for some } \lambda>0 .
$$

Then, for the ball $\mathbb{B}(y, R) \subset \Omega$ we have

$$
\int_{r}^{R} \frac{\mathrm{d} s}{s\left(f_{\partial \mathbb{B}(y, s)} \beta(x)^{q} \mathrm{~d} \sigma\right)^{\gamma / q}} \geqslant F\left(R / e^{3}\right)-F(r),
$$

whenever $0<r<R / e^{3}$. Here

$$
F(s)= \begin{cases}\frac{\lambda^{\gamma}}{n(\gamma-1)} \log ^{-\gamma+1}\left(\frac{e^{t_{0}} K}{\omega_{n-1} s^{n}}\right) & \text { if } \gamma \neq 1 \\ -\frac{\lambda}{n} \log \log \left(\frac{e^{t_{0}} K}{\omega_{n-1} s^{n}}\right) & \text { if } \gamma=1\end{cases}
$$

where $t_{0}=\max ((q-1) / \lambda,(\gamma-1) / \lambda)$.

Proof. For simplicity, we write $\mathbb{B}_{s}=\mathbb{B}(y, s)$. Let $i_{R}$ and $i_{r}$ be integers such that $\log R-1<i_{R} \leqslant \log R$ and $\log r \leqslant i_{r}<\log r+1$. We have

$$
\int_{r}^{R} \frac{\mathrm{d} s}{s\left(f_{\partial \mathbb{B}_{s}} \beta(x)^{q} \mathrm{~d} \sigma\right)^{\gamma / q}} \geqslant \sum_{i=i_{r}}^{i_{R}-1} \int_{e^{i}}^{e^{i+1}} \frac{\mathrm{d} s}{s\left(f_{\partial \mathbb{B}_{s}} \beta(x)^{q} \mathrm{~d} \sigma\right)^{\gamma / q}} .
$$

Next, we estimate each integral on the right hand side of (2.3) in the following way. Changing the variable by the formula $s=e^{t}$, we obtain

$$
\int_{e^{i}}^{e^{i+1}} \frac{\mathrm{d} s}{s\left(f_{\partial \mathbb{B}_{s}} \beta(x)^{q} \mathrm{~d} \sigma\right)^{\gamma / q}}=\int_{i}^{i+1} \frac{\mathrm{d} t}{\left(f_{\partial \mathbb{B}_{e^{t}}} \beta(x)^{q} \mathrm{~d} \sigma\right)^{\gamma / q}} .
$$

Since the function $\tau \rightarrow 1 / \tau$ is convex on $(0, \infty)$, Jensen's inequality yields

$$
\int_{i}^{i+1} \frac{\mathrm{d} t}{\left(f_{\partial \mathbb{B}_{e^{t}}} \beta(x)^{q} \mathrm{~d} \sigma\right)^{\gamma / q}} \geqslant\left[\int_{i}^{i+1}\left(f_{\partial \mathbb{B}_{e^{t}}} \beta(x)^{q} \mathrm{~d} \sigma\right)^{\gamma / q} \mathrm{~d} t\right]^{-1} .
$$

Combining (2.3), (2.4) and (2.5), we arrive at

$$
\int_{r}^{R} \frac{\mathrm{d} s}{s\left(f_{\partial \mathbb{B}_{s}} \beta(x)^{q} \mathrm{~d} \sigma\right)^{\gamma / q}} \geqslant \sum_{i=i_{r}}^{i_{R}-1}\left[\int_{i}^{i+1}\left(f_{\partial \mathbb{B}_{e^{t}}} \beta(x)^{q} \mathrm{~d} \sigma\right)^{\gamma / q} \mathrm{~d} t\right]^{-1} .
$$


We continue to estimate the right hand side of (2.6). For that, we define the following auxiliary function

$$
\tilde{\beta}(x)= \begin{cases}\beta(x) & \text { if } \beta(x)>t_{0} \\ t_{0} & \text { if } \beta(x) \leqslant t_{0}\end{cases}
$$

where $t_{0}=\max ((q-1) / \lambda,(\gamma-1) / \lambda) \geqslant 0$, defined as in the lemma. Note that the function $t \rightarrow \exp \left(\lambda t^{1 / q}\right)$ is convex for $t \geqslant(q-1)^{q} / \lambda^{q}$. Again, Jensen's inequality gives

$$
\begin{aligned}
\left(f_{\partial \mathbb{B}_{e^{t}}} \beta(x)^{q} \mathrm{~d} \sigma\right)^{1 / q} & \leqslant\left(f_{\partial \mathbb{B}_{e^{t}}} \tilde{\beta}(x)^{q} \mathrm{~d} \sigma\right)^{1 / q} \\
& \leqslant \frac{1}{\lambda} \log \left(f_{\partial \mathbb{B}_{e^{t}}} \exp (\lambda \tilde{\beta}(x)) \mathrm{d} \sigma\right)
\end{aligned}
$$

Thus for each $i=i_{r}, \ldots, i_{R}-1$,

$$
\begin{aligned}
& \int_{i}^{i+1}\left(f_{\left.\partial \mathbb{B}_{e^{t}} \beta(x)^{q} \mathrm{~d} \sigma\right)^{\gamma / q} \mathrm{~d} t} \leqslant \frac{1}{\lambda \gamma} \int_{i}^{i+1} \log ^{\gamma}\left(f_{\partial \mathbb{B}_{e^{t}}} \exp (\lambda \tilde{\beta}(x)) \mathrm{d} \sigma\right) \mathrm{d} t\right. \\
& \leqslant \frac{1}{\lambda \gamma} \log ^{\gamma}\left(\int_{i}^{i+1} f_{\partial \mathbb{B}_{e^{t}}} \exp (\lambda \tilde{\beta}(x)) \mathrm{d} \sigma \mathrm{d} t\right) \\
&=\frac{1}{\lambda \gamma} \log ^{\gamma}\left(\int_{e^{i}}^{e^{i+1}} \frac{1}{s} f_{\partial \mathbb{B}_{s}} \exp (\lambda \tilde{\beta}(x)) \mathrm{d} \sigma \mathrm{d} s\right) \\
& \leqslant \frac{1}{\lambda^{\gamma}} \log ^{\gamma}\left(\frac{e^{t_{0}} K}{\omega_{n-1} e^{n i}}\right),
\end{aligned}
$$

where in the second inequality above, we used once more Jensen's inequality. Note that the function $t \rightarrow \exp \left(t^{1 / \gamma}\right)$ is convex for $t \geqslant[\max (\gamma-1,0)]^{\gamma}$. Putting the above estimates together we conclude that

$$
\begin{aligned}
\int_{r}^{R} \frac{\mathrm{d} s}{s\left(f_{\partial \mathbb{B}_{s}} \beta(x)^{q} \mathrm{~d} \sigma\right)^{\gamma / q}} & \geqslant \sum_{i=i_{r}}^{i_{R}-1} \lambda^{\gamma} \log ^{-\gamma}\left(\frac{e^{t_{0}} K}{\omega_{n-1} e^{n i}}\right) \\
& \geqslant \lambda^{\gamma} \int_{i_{r}-1}^{i_{R}-2} \log ^{-\gamma}\left(\frac{e^{t_{0}} K}{\omega_{n-1} e^{n t}}\right) \mathrm{d} t \\
& \geqslant \lambda^{\gamma} \int_{r}^{R / e^{3}} s^{-1} \log ^{-\gamma}\left(\frac{e^{t_{0}} K}{\omega_{n-1} s^{n}}\right) \mathrm{d} s
\end{aligned}
$$

which proves the lemma. 


\section{Proof of Theorem 1.2}

A very powerful method when dealing with continuity properties of functions is furnished by notion of monotonicity, which goes back to Lebesgue [8] in 1907. Monotonicity for a continuous function $u$ in a domain $\Omega$ simply means that

$$
\operatorname{osc}(u, \mathbb{B}) \leqslant \operatorname{osc}(u, \partial \mathbb{B})
$$

for every ball $\mathbb{B} \subset \Omega$. It turns out that monotonicity can be defined without the continuity assumption. The following definition is due to Manfredi [9].

Definition 3.1. A real valued function $v \in W^{1,1}(\Omega)$ is said to be weakly monotone if for every ball $\mathbb{B} \subset \Omega$ and all constants $m \leqslant M$ such that

$$
(v-M)^{+}-(m-v)^{+} \in W_{\circ}^{1,1}(\mathbb{B})
$$

we have

$$
m \leqslant v(x) \leqslant M
$$

for almost every $x \in \mathbb{B}$.

As usually, the space $W_{\circ}^{1,1}(\mathbb{B})$ is the completion of $C_{\circ}^{\infty}(\mathbb{B})$ in $W^{1,1}(\mathbb{B})$.

It is known that any weak solution of equation (1.1) is weakly monotone in domain $\Omega^{\prime}$ compactly contained in $\Omega$ under the assumption of Theorem 1.2. This simply follows from using $(u-M)^{+}$and $(m-u)^{+}$as a test function in (1.4) and applying the lower bound (1.2) for the matrix $A(x)$. These are legitimate test functions due to the assumption (1.3) and the following point-wise estimate

$$
\langle A(x) \nabla u, \nabla \phi\rangle \leqslant|\nabla \phi| \sqrt{\langle A(x) \nabla u, \nabla u\rangle} .
$$

Here we also used the upper bound at (1.2). Monotonicity allows us to show the following oscillation estimate for a solution $u$. Let $\mathbb{B}\left(x_{\circ}, r\right)$ be a ball compactly contained in $\Omega$.

Lemma 3.2. Under the hypothesis of Theorem 1.2, we have

$$
|u(x)-u(y)|^{2} \leqslant 64 \pi \log \left(\frac{K}{\pi r^{2}}\right) \int_{\mathbb{B}\left(x_{\circ}, r\right)}\langle A(z) \nabla u, \nabla u\rangle \mathrm{d} z
$$

for almost every $x, y \in \mathbb{B}\left(x_{\circ},\left(\frac{2 \pi}{e^{13} K}\right)^{1 / 2} r^{2}\right)$.

Proof. Actually, we will prove the estimate (3.4) under slightly weaker assumptions than are stated in the lemma, namely it is enough to require that the function $\sqrt{\alpha(x)}$ is exponentially integrable, i.e. (1.8) is valid for $\lambda=1$. Fix a ball $\mathbb{B}_{r}=\mathbb{B}\left(x_{\circ}, r\right)$ which is compactly contained in $\Omega$. Monotonicity of $u$ gives that for almost every $t \in(0, r)$ we have

$$
|u(x)-u(y)| \leqslant \int_{\partial \mathbb{B}_{t}}|\nabla u(z)| \mathrm{d} \sigma
$$


for almost every $x, y \in \mathbb{B}_{t}$. Therefore, by the assumption (1.2) and Hölder's inequality we arrive at

$$
|u(x)-u(y)|^{2} \leqslant \int_{\partial \mathbb{B}_{t}} \alpha(z) \mathrm{d} \sigma \int_{\partial \mathbb{B}_{t}}\langle A(z) \nabla u, \nabla u\rangle \mathrm{d} \sigma .
$$

Thus

$$
\frac{|u(x)-u(y)|^{2}}{t f_{\partial \mathbb{B}_{t}} \alpha(z) \mathrm{d} \sigma} \leqslant 2 \pi \int_{\partial \mathbb{B}_{t}}\langle A(z) \nabla u, \nabla u\rangle \mathrm{d} \sigma .
$$

We integrate both sides of the above inequality with respect to the variable $t$ over $\left(r^{\prime}, r\right)$, where $r^{\prime}<r / e^{3}$ will be chosen later. We have for almost every $x, y \in \mathbb{B}_{r^{\prime}}$,

$$
|u(x)-u(y)|^{2} \int_{r^{\prime}}^{r} \frac{\mathrm{d} t}{t f_{\partial \mathbb{B}_{t}} \alpha(z) \mathrm{d} \sigma} \leqslant 2 \pi \int_{\mathbb{B}_{r}}\langle A(z) \nabla u, \nabla u\rangle \mathrm{d} z .
$$

To estimate from below the integral on the left hand side of the above inequality, we apply Lemma 2.1 with $\beta(z)=\sqrt{\alpha(z)}, q=2, \gamma=2$ and $\lambda=1$. We arrive at

$$
\left[F\left(r / e^{3}\right)-F\left(r^{\prime}\right)\right]|u(x)-u(y)|^{2} \leqslant 2 \pi \int_{\mathbb{B}_{r}}\langle A(z) \nabla u, \nabla u\rangle \mathrm{d} z,
$$

where

$$
F(s)=\frac{1}{2} \log ^{-1}\left(\frac{e K}{2 \pi s^{2}}\right) .
$$

Now the lemma follows by choosing $r^{\prime}<r / e^{3}$ such that $F\left(r^{\prime}\right)=F\left(r / e^{3}\right) / 2$, that is, $r^{\prime}=\left(\frac{2 \pi}{e^{13} K}\right)^{1 / 2} r^{2}$, and employing the elementary inequality

$$
\log \left(\frac{e^{7} K}{2 \pi r^{2}}\right) \leqslant 8 \log \left(\frac{K}{\pi r^{2}}\right)
$$

Here we employed the fact $\frac{K}{\pi r^{2}} \geqslant e$. This completes the proof of Lemma 3.2.

Remark 3.3. If the function $\alpha$ satisfies the exponential integrability condition at (1.7), that is,

$$
\int_{\Omega} \exp (\lambda \alpha(x)) \mathrm{d} x<\infty, \quad \text { for some } \lambda>0,
$$

then, as mentioned in the introduction, any weak solution of the equation (1.1) is continuous. Indeed, applying Lemma 2.1 to the inequality (3.8) with $\beta(x)=$ $\alpha(x), q=1, \gamma=1$ we find that the solution is continuous and it has $(\log \log )^{\frac{1}{2}}$ type modulus of continuity. 
Lemma 3.4. Under the hypothesis of Theorem 1.2, for any $1 \leqslant v<\lambda$, there exists a constant $C=C(\lambda, v, K, R)$ such that

$$
\int_{\mathbb{B}_{r}}\langle A(x) \nabla u, \nabla u\rangle \mathrm{d} x \leqslant C \log ^{-v}\left(\frac{K}{\pi r^{2}}\right) \int_{\mathbb{B}_{R}}\langle A(x) \nabla u, \nabla u\rangle \mathrm{d} x
$$

whenever $\mathbb{B}_{R}=\mathbb{B}\left(x_{\circ}, R\right)$ is compactly contained in $\Omega$ and $0<r<R / e^{3}$.

A crucial tool to establish the sharp decay estimate in Lemma 3.4 is to apply the Poincaré inequality with the sharp constant. Let $\mathbb{I}$ be the interval $[0,2 \pi]$ in $\mathbb{R}$ and $p \geqslant 1$. If $\omega \in W^{1, p}(\mathbb{I}, \mathbb{R})$, then

$$
\left(f_{\mathbb{I}}\left|\omega(x)-\omega_{\mathbb{I}}\right|^{2} \mathrm{~d} x\right)^{\frac{1}{2}} \leqslant A_{p}\left(f_{\mathbb{I}}\left|\omega^{\prime}(x)\right|^{p} \mathrm{~d} x\right)^{\frac{1}{p}} .
$$

Here $\omega_{\mathbb{I}}=f_{\mathbb{I}} \omega(x) \mathrm{d} x$ is the average of $\omega$ over the interval $\mathbb{I}$. The sharp constant $A_{p}$ is well-known in the literature, see for example [19]. Instead of giving the precise formula for $A_{p}$ we only recall two facts that will play a crucial role. The first thing that we will employ is continuity of $A_{p}$ with respect $p$. Secondly, when $p=2$, the inequality (3.10), also known as Wirtinger's inequality in the literature [5], holds with the constant $A_{2}=1$. More precisely, in our case $\omega \in W^{1, p}(\mathbb{I}, \mathbb{R})$ for all $p<2$ and we will only use the fact that the constant $A_{p}$ approaches 1 as $p \rightarrow 2-$.

Proof of Lemma 3.4. Without loss of generality, we can assume that the origin lies in $\Omega$ and prove the estimate (3.9) for balls centered at the origin. We write $\mathbb{B}_{s}=\mathbb{B}(0, s)$ for all $0<s<\operatorname{dist}(0, \partial \Omega)$. Our starting point is to verify the inequality

$$
\int_{\mathbb{B}_{s}}\langle A(x) \nabla u, \nabla u\rangle \mathrm{d} x \leqslant \int_{\partial \mathbb{B}_{s}}\left|\left\langle A(x) \nabla u, \eta_{x}\right\rangle(u-c)\right| \mathrm{d} \sigma
$$

for almost every $0<s<\operatorname{dist}(0, \partial \Omega)$. Here $\eta_{x}$ stands for the normal unit vector to $\partial \mathbb{B}_{s}$ at the point $x$ and $c$ is a constant. Later, we will choose the constant $c$ to be the average of $u$ over the 1 -dimensional sphere $\partial \mathbb{B}_{s}$.

Proof of (3.11). Fix a ball $\mathbb{B}_{s}$. For sufficiently small $\epsilon \in(0, s / 2)$ we define an auxiliary Lipschitz function by the rule

$$
\Gamma_{\epsilon}(x)= \begin{cases}\frac{s-|x|}{\epsilon} & \text { if } s-\epsilon \leqslant|x|<s, \\ 1 & \text { if }|x|<s-\epsilon .\end{cases}
$$

Using the function $\varphi_{\epsilon}(x)=(u(x)-c) \Gamma_{\epsilon}(x)$ as a test-function in (1.4), we have

$$
\int_{\mathbb{B}_{s}}\left\langle A(x) \nabla u(x), \nabla \varphi_{\epsilon}(x)\right\rangle \mathrm{d} x=0 .
$$


Notice that $\varphi_{\epsilon}$ is an admissible test-function due to the structure condition (1.2) and the estimate (3.4). By the product rule and the fact $\left|\nabla \Gamma_{\epsilon}\right| \leqslant 1 / \epsilon$ we have

$$
\begin{aligned}
\int_{\mathbb{B}_{s-\epsilon}}\langle A(x) \nabla u, \nabla u\rangle \mathrm{d} x & \leqslant \frac{1}{\epsilon} \int_{\mathbb{B}_{s} \backslash \mathbb{B}_{s-\epsilon}}\left|\left\langle A(x) \nabla u, \eta_{x}\right\rangle(u-c)\right| \mathrm{d} x \\
& =\frac{1}{\epsilon} \int_{s-\epsilon}^{s} \int_{\partial \mathbb{B}_{r}}\left|\left\langle A(x) \nabla u, \eta_{x}\right\rangle(u-c)\right| \mathrm{d} \sigma \mathrm{d} r .
\end{aligned}
$$

The claim (3.11) follows by letting $\epsilon \rightarrow 0$.

Our second goal is to estimate the right hand side of (3.11) and verify for $1 \leqslant p<2$,

$$
\begin{aligned}
& \int_{\partial \mathbb{B}_{s}}\left|u-u_{\partial \mathbb{B}_{s}}\right|\left|\left\langle A(x) \nabla u, \eta_{x}\right\rangle\right| \mathrm{d} \sigma \leqslant \frac{A_{p}}{2} s\left(f_{\partial \mathbb{B}_{s}}[\alpha(x)]^{\frac{p}{2-p}} \mathrm{~d} \sigma\right)^{\frac{2-p}{2 p}} \\
& \times \int_{\partial \mathbb{B}_{s}}\langle A(x) \nabla u, \nabla u\rangle \mathrm{d} \sigma,
\end{aligned}
$$

where $u_{\partial \mathbb{B}_{s}}$ stands for the integral average of $u$ over the sphere $\partial \mathbb{B}_{s}$ and $A_{p}$ is the sharp constant in Poincaré's inequality (3.10). A crucial property of the constant $A_{p}$ for us is that it approaches to 1 as $p \rightarrow 2-$.

Proof of (3.15). Applying Hölder's inequality we obtain that

$$
\begin{aligned}
\int_{\partial \mathbb{B}_{s}}\left|u-u_{\partial \mathbb{B}_{s}}\right|\left|\left\langle A(x) \nabla u, \eta_{x}\right\rangle\right| \mathrm{d} \sigma \leqslant & \left(\int_{\partial \mathbb{B}_{s}} \mid u-u_{\left.\left.\partial \mathbb{B}_{s}\right|^{2} \mathrm{~d} \sigma\right)^{\frac{1}{2}}}\right. \\
& \times\left(\int_{\partial \mathbb{B}_{s}}\left|\left\langle A(x) \nabla u, \eta_{x}\right\rangle\right|^{2} \mathrm{~d} \sigma\right)^{\frac{1}{2}} .
\end{aligned}
$$

For fixed $1 \leqslant p<2$, the sharp form of the Poincaré inequality (3.10) gives

$$
\left(f_{\partial \mathbb{B}_{s}}\left|u-u_{\partial \mathbb{B}_{s}}\right|^{2} \mathrm{~d} \sigma\right)^{\frac{1}{2}} \leqslant A_{p} s\left(f_{\partial \mathbb{B}_{s}}\left|u_{T}\right|^{p} \mathrm{~d} \sigma\right)^{\frac{1}{p}}
$$

where $u_{T}$ stands for the tangential derivative of the function $u$. Notice that these inequalities together with the following point-wise estimate

$$
\left\langle A(x) \nabla u, \eta_{x}\right\rangle^{2} \leqslant\langle A(x) \nabla u, \nabla u\rangle\left\langle A(x) \eta_{x}, \eta_{x}\right\rangle
$$

and the assumption (1.2) lead to the desired inequality (3.15) with the constant $A_{p}$ in the place of $A_{p} / 2$. In order to obtain the desired estimate (3.15), i.e. to gain the constant 1/2, we will follow the idea of Piccinini and Spagnolo in [17] to explore the special structure of the product

$$
\left(\int_{\partial \mathbb{B}_{s}}\left|u_{T}\right|^{p} \mathrm{~d} \sigma\right)^{\frac{1}{p}}\left(\int_{\partial \mathbb{B}_{s}}\left|\left\langle A(x) \nabla u, \eta_{x}\right\rangle\right|^{2} \mathrm{~d} \sigma\right)^{\frac{1}{2}} .
$$


The unit vector $\eta_{x}$ and the tangential derivative of $u$ in this product suggest to write the integrals in terms of polar coordinates. Let $x_{1}=\rho \cos \theta$ and $x_{2}=\rho \sin \theta$ be the polar coordinate transformation. We denote the normal derivative of the function $u$ by

$$
u_{N}=u_{\rho}=\frac{\partial u}{\partial \rho}
$$

and the tangential derivative by

$$
u_{T}=\frac{u_{\theta}}{\rho}=\frac{1}{\rho} \frac{\partial u}{\partial \theta} .
$$

Also we denote the spherical gradient by $\nabla_{S} u=\left(u_{N}, u_{T}\right)$. Using these notation we can write

$$
\left\langle A \nabla u, \eta_{x}\right\rangle=\left\langle A \mathcal{O} \nabla_{S} u, \mathcal{O} e_{1}\right\rangle
$$

where $\mathcal{O}$ stands for the orthogonal matrix

$$
\mathcal{O}=\left(\begin{array}{cc}
\cos \theta & -\sin \theta \\
\sin \theta & \cos \theta
\end{array}\right)
$$

and $e_{1}=(1,0)$. Briefly, we denote the symmetric matrix

$$
\mathcal{O}^{T}(x) A(x) \mathcal{O}(x) \text { by } B(x)=\left(\begin{array}{ll}
b_{11}(x) & b_{12}(x) \\
b_{12}(x) & b_{22}(x)
\end{array}\right)
$$

and, therefore, we have

$$
\left\langle B(x) \nabla_{S} u, e_{1}\right\rangle=\left\langle A(x) \nabla u, \eta_{x}\right\rangle
$$

and

$$
\left\langle B(x) \nabla_{S} u, \nabla_{S} u\right\rangle=\langle A(x) \nabla u, \nabla u\rangle .
$$

Since the matrix $B(x)$ has the same eigenvalues as the original matrix $A(x)$, the assumption (1.2) gives

$$
\left\{\begin{array}{l}
0<\frac{1}{\alpha(x)} \leqslant b_{11}(x) \leqslant 1 \\
0<\frac{1}{\alpha(x)} \leqslant b_{22}(x)-\frac{b_{12}^{2}(x)}{b_{11}(x)} \leqslant 1
\end{array}\right.
$$

for almost every $x \in \Omega$. Now we are ready to estimate the product (3.19). Combining (3.25) with the inequality $\sqrt{b_{11}(x)} \leqslant 1$ at (3.27), we find that

$$
\begin{aligned}
\left\langle A(x) \nabla u, \eta_{x}\right\rangle^{2} & =\left\langle B(x) \nabla_{S} u, e_{1}\right\rangle^{2}=\left(b_{11}(x) u_{N}(x)+b_{12}(x) u_{T}(x)\right)^{2} \\
& \leqslant\left(\sqrt{b_{11}(x)} u_{N}(x)+\frac{b_{12}(x)}{\sqrt{b_{11}(x)}}(x) u_{T}(x)\right)^{2}
\end{aligned}
$$


Multiplying the second estimate at (3.27) by $\alpha(x) u_{T}^{2}$ and then raising it to the power $p / 2$ we have

$$
\left|u_{T}\right|^{p} \leqslant\left[\alpha(x) u_{T}^{2}\left(b_{22}(x)-\frac{\left[b_{12}(x)\right]^{2}}{b_{11}(x)}\right)\right]^{\frac{p}{2}} .
$$

Applying Hölder's inequality for (3.29) we are led to the following estimate

$$
\left(\int_{\partial \mathbb{B}_{s}}\left|u_{T}\right|^{p}\right)^{\frac{1}{p}} \leqslant\left(\int_{\partial \mathbb{B}_{s}}[\alpha(x)]^{\frac{p}{2-p}}\right)^{\frac{2-p}{2 p}}\left(\int_{\partial \mathbb{B}_{s}}\left(b_{22}(x)-\frac{\left[b_{12}(x)\right]^{2}}{b_{11}(x)}\right) u_{T}^{2}\right)^{\frac{1}{2}} .
$$

Combining the estimates (3.28) and (3.30) with the elementary point-wise inequality $\sqrt{a b} \leqslant \frac{1}{2}(a+b)$, for $a, b \geqslant 0$, we arrive at

$$
\begin{aligned}
& \left(\int_{\partial \mathbb{B}_{S}}\left|u_{T}\right|^{p}\right)^{\frac{1}{p}}\left(\int_{\partial \mathbb{B}_{S}}\left|\left\langle A(x) \nabla u, \eta_{x}\right\rangle\right|^{2}\right)^{\frac{1}{2}} \\
& \quad \leqslant \frac{1}{2}\left(\int_{\partial \mathbb{B}_{S}}[\alpha(x)]^{\frac{p}{2-p}}\right)^{\frac{2-p}{2 p}}\left(\int_{\partial \mathbb{B}_{S}}\left\langle B(x) \nabla_{S} u, \nabla_{S} u\right\rangle\right) .
\end{aligned}
$$

Finally, the claim (3.15) follows from the estimate (3.31) together with (3.26) and Poincaré's inequality (3.17).

Putting together (3.11) and (3.15) that we proved, we are led to the following elementary differential equation

$$
\frac{\mathrm{d}}{\mathrm{d} s}\left(\log \int_{\mathbb{B}_{s}}\langle A(x) \nabla u, \nabla u\rangle \mathrm{d} x\right) \geqslant \frac{2}{A_{p}} s^{-1}\left(f_{\partial \mathbb{B}_{s}}[\alpha(x)]^{\frac{p}{2-p}} \mathrm{~d} \sigma\right)^{\frac{p-2}{2 p}} .
$$

First, we integrate both sides of the above inequality with respect to $s$ over $(r, R)$ for $r<R / e^{3}$. Second, we apply Lemma 2.1 with $\beta(x)=\sqrt{\alpha(x)}, q=2 p /(2-$ $p), \gamma=1$ to obtain that

$$
\log \left(\frac{\int_{\mathbb{B}_{R}}\langle A(x) \nabla u, \nabla u\rangle \mathrm{d} x}{\int_{\mathbb{B}_{r}}\langle A(x) \nabla u, \nabla u\rangle \mathrm{d} x}\right) \geqslant \frac{2}{A_{p}}\left(F\left(R / e^{3}\right)-F(r)\right),
$$

where

$$
F(s)=-\frac{\lambda}{2} \log \log \left(\frac{e^{t_{0}} K}{2 \pi s^{2}}\right)
$$

and $t_{0}=(3 p-2) /(2-p) \lambda$. At this moment, we fix $p$. We choose $p$ so close to 2 , that $\lambda / A_{p} \geqslant v$ and $t_{0} \geqslant 1$. The second requirement is easily fulfilled. Also the first 
one is possible since $v<\lambda$ and $A_{p} \rightarrow 1$ as $p \rightarrow 2-$. The desired decay estimate (3.9) on the energy of $u$ then follows from (3.32) and the elementary inequality

$$
\log \log \left(\frac{e^{t_{0}} K}{2 \pi s^{2}}\right) \geqslant \log \log \left(\frac{K}{\pi s^{2}}\right) .
$$

This finishes the proof of Lemma 3.4.

Proof of Theorem 1.2. Theorem 1.2 simply follows combining Lemma 3.2 with Lemma 3.4.

\section{References}

[1] E. De Giorgi, Sulla differenziabilità e l'analiticità delle estremali degli integrali multipli regolari, Mem. Accad. Sci. Torino Cl. Sci. Fis. Mat. Natur. 3 (1957), 25-43.

[2] E. De GIORgI, Congetture sulla continuità delle soluzioni di equazioni lineari ellittiche autoaggiunte a coefficienti illimitati, Unpublished, 1995.

[3] B. Franchi, R. Serapioni and F. CASSANo, Irregular solutions of linear degenerate elliptic equations Potential Anal. 9 (1998), 201-216.

[4] D. Gilbarg and N. S. Trudinger, "Elliptic Partial Differential Equations of Second Order" 2nd ed., Springer-Verlag, New York, 1983.

[5] G. H. Hardy, J. E. LitTlewood and G. Polya, "Inequalities", 2nd ed., Cambridge University Press, Cambridge, 1952.

[6] T. IWANIEC, P. Koskela and J. ONNINEN, Mappings of finite distortion: monotonicity and continuity, Invent. Math. 144 (2001), 507-531.

[7] O. A. LAdyzhenskaya and N. N. URAL'tseva, "Linear and Quasilinear Elliptic Equations", Academic Press, New York, 1968.

[8] H. Lebesgue, Sur le problème de Dirichlet. Rend. Circ. Mat. Palermo 27 (1907), 371-402.

[9] J. J. MANFREDI, Weakly monotone functions, J. Geom. Anal. 4 (1994), 393-402.

[10] N. G. MEYERS, An $L^{p}$-estimate for the gradient of solutions of second order elliptic divergence equations, Ann. Scuola Norm. Sup. Pisa Cl. Sci. (3) 17 (1963), 189-206.

[11] C. B. Morrey, On the solutions of quasi-linear elliptic partial differential equations, Trans. Amer. Math. Soc. 43 (1938), 126-166.

[12] C. B. MorRey, Multiple integral problems in the calculus of variations and related topics. Univ. California Publ. Math. (N. S.) 1 (1943), 1-130.

[13] J. MOSER, A new proof of De Giorgi's theorem concerning the regularity problem for elliptic differential equations, Comm. Pure Appl. Math. 13 (1960), 457-468.

[14] J. Moser, On Harnack's theorem for elliptic differential equations, Comm. Pure Appl. Math. 14 (1961), 577-591.

[15] J. NASH, Continuity of solutions of elliptic and parabolic equations, Amer. J. Math. 80 (1958), 931-954.

[16] J. ONNINEN and X. ZHONG, A note on mappings of finite distortion: the sharp modulus of continuity, Michigan Math. J. 53 (2005), 329-335.

[17] L. C. PICCININI and S. SPAGNOLO, On the Hölder continuity of solutions of second order elliptic equations in two variables, Ann. Scuola Norm. Sup. Pisa Cl. Sci. (3) 26 (1972), 391402.

[18] J. SERRIN, On the strong maximum principle for quasilinear second order differential inequalities, J. Funct. Anal. 5 (1970), 184-193.

[19] G. Talenti, Best constant in Sobolev inequality, Ann. Mat. Pura Appl. (4) 110 (1976), 353-372. 
[20] N. S. TRUdinger, On Harnack type inequalities and their application to quasilinear, elliptic equations, Comm. Pure Appl. Math. 20 (1967), 721-747.

[21] N. S. TRUdinger, On the regularity of generalized solutions of linear, non-uniformly elliptic equations, Arch. Rational Mech. Anal. 42 (1971), 51-62.

[22] K. O. WidmAn, On the Hölder continuity of solutions of elliptic partial differential equations in two variables with coefficients in $L^{\infty}$, Comm. Pure Appl. Math. 22 (1969), 669-682.

Department of Mathematics

Syracuse University

Syracuse, NY 13244, USA

jkonnine@syr.edu

Department of Mathematics and Statistics

University of Jyväskylä

P.O. Box 35 (MaD), Fin-40014

University of Jyväskylä, Finland zhong@maths.jyu.fi 